\title{
The heavier the better: how to constrain mass ratios and spins of high-mass neutron-star mergers
}

\author{
Elias R. Most ${ }^{1 \star}$, Lukas R. Weih ${ }^{1}$, Luciano Rezzolla ${ }^{1,2}$ \\ ${ }^{1}$ Institut für Theoretische Physik, Goethe Universität Frankfurt am Main, Germany \\ 2 School of Mathematics, Trinity College, Dublin 2, Ireland
}

Accepted XXX. Received YYY; in original form ZZZ

\begin{abstract}
The first binary neutron-star merger event, GW170817, and its bright electromagnetic counterpart have provided a remarkable amount of information. By contrast, the second event, GW190425, with $M_{\mathrm{tot}}=3.4_{-0.1}^{+0.3} M_{\odot}$ and the lack of an electromagnetic counterpart, has hardly improved our understanding of neutron-star physics. While GW190425 is compatible with a scenario in which the merger has lead to a prompt collapse to a black hole and little ejected matter to power a counterpart, determining the mass ratio and the effective spin $\tilde{\chi}$ of the binary remains difficult. This is because gravitational waveforms cannot yet well constrain the component spins of the binary. However, since the mass of GW190425 is significantly larger than the maximum mass for nonrotating neutron stars, $M_{\mathrm{TOV}}$, the mass ratio $q$ cannot be too small, as the heavier star would not be gravitationally stable. Making use of universal relations and a large number of equations of state, we provide limits in the $(\tilde{\chi}, q)$ plane for GW190425, namely: $q_{\min } \geq 0.38$ and $\tilde{\chi}_{\max } \leq 0.20$, assuming $M_{\text {tot }} \simeq 3.4 M_{\odot}$. Finally, we show how future observations of high-mass binaries can provide a lower bound on $M_{\mathrm{Tov}}$.
\end{abstract}

Key words: gravitational waves - stars:neutron

\section{INTRODUCTION}

After the first detection of gravitational waves from a binary neutron star system (The LIGO Scientific Collaboration \& The Virgo Collaboration 2017), in April of 2019 a second event, GW190425, was recorded by the LIGO-VIRGO collaboration (Abbott et al. 2020). For the latter, however, no electromagnetic counterpart could be detected (Coughlin et al. 2020), which is likely due to the merger's large distance $\left(159_{-71}^{+69} \mathrm{Mpc}\right)$, the corresponding broad sky localisation, or a sign for prompt collapse of the merger's remnant (Foley et al. 2020). Furthermore, the total binary mass of $M_{\mathrm{tot}}=3.4_{-0.1}^{+0.3} M_{\odot}$ is unexpectedly high and in contradiction to observed Galactic populations of neutron stars in binary systems, which have masses of 2.5-2.89 $M_{\odot}$ (see, e.g., Farrow et al. 2019), thus challenging our knowledge of its formation channel (Romero-Shaw et al. 2020; Safarzadeh et al. 2020). Combined with only a very weak upper bound on the tidal deformability $\tilde{\Lambda}<1000$, these uncertainties have prevented significant constraints on the equation of state (EOS). Indeed, even the possibility of classifying this event as a black hole-neutron star merger is still possible - albeit less likely (Kyutoku et al. 2020; Han et al. 2020). This is to be contrasted with the multimessenger event GW170817, which - thanks to the clear electromagnetic counterpart and strong limits on the tidal deformability $\tilde{\Lambda}$ - has led to a number of constraints on the EOS of nuclear matter (see Margalit \& Metzger 2017; Rezzolla et al. 2018; Ruiz et al. 2018; Annala et al. 2018; Radice et al. 2018; Most et al. 2018; Tews et al. 2018; De et al. 2018; Abbott et al. 2018; Montaña et al. 2019; Shibata et al. 2019; Koeppel et al. 2019, for an incomplete list). However, the unusually high mass of GW190425 does allow us to constrain the effective spin, $\tilde{\chi}$, and mass ratio, $q$, of the system. To establish such constraints under the assumption of GW190425 having been a neutron-star binary merger, we make use of universal relations (Breu \& Rezzolla 2016) for the critical mass and compactness of rotating neutron stars and combine these relations with a large, publicly available dataset of $O\left(10^{6}\right)$ EOSs consistent with the aforementioned constrains derived from GW170817 (Weih et al. 2019a). In this way, we can not only constrain the parameter space of GW190425, but also provide new and robust constraints for $q$ and $\tilde{\chi}$ that can be used to rule out the most extreme orbital configurations in future gravitationalwave events of neutron-star binaries even in the absence of an electromagnetic counterpart.

^ emost@itp.uni-frankfurt.de

(C) 2020 The Authors 


\section{THE BROADBRUSH PICTURE}

To illustrate our method for constraining the allowed parameter space for the mass ratio $q$ and the effective dimensionless spin $\tilde{\chi}$ [see (1) and (3) for definitions] for high-mass mergers we make use of the schematic diagram shown in Fig. 1. We recall that gravitational-wave detections measure the chirp mass with high precision and thus provide an accurate estimate for the total mass $M_{\mathrm{tot}}$ of the system, whereas the mass ratio $q$ and dimensionless spin $\tilde{\chi}$ are much less constrained. Consider therefore a gravitational-wave detection and an inferred $(\tilde{\chi}, q)$ allowed band (shaded-blue area in Fig. 1). Such a band will likely include a region of allowed solutions corresponding to either nonrotating or uniformly rotating neutron stars (green-shaded area), and an excluded region in which no such configuration can be found (redshaded area). More precisely, given a neutron-star binary with total mass $M_{\text {tot }}$ and mass ratio $q:=m_{2} / m_{1} \leq 1$, the component masses of the binary $m_{1}$ (primary) and $m_{2}$ (secondary) are given respectively by

$m_{1}=\frac{1}{1+q} M_{\mathrm{tot}}, \quad m_{2}=\frac{q}{1+q} M_{\mathrm{tot}}$.

Hence, assuming some value for the mass ratio $q$ we can then infer the component masses $m_{1}$ and $m_{2}$ of the binary using Eqs. (1), so long as they correspond to gravitationally stable configurations. If the more massive (primary) star is nonorotating, then it must have a mass smaller than the maximum mass for nonrotating configurations, i.e., $m_{1}<$ $M_{\mathrm{TOV}}\left(M_{\mathrm{TOV}}\right.$ is marked with black circles for different EOSs). On the other hand, if it is rotationally supported with spin $S_{1}$, then it must have a mass smaller than the "critical mass" $M_{\text {crit }}\left(S_{1}\right)$, that is, the maximum mass allowed for such a spin, i.e., $m_{1}<M_{\text {crit }}\left(S_{1}\right)$ ( $M_{\text {crit }}$ is marked with black stars). Such a critical mass follows the neutral-stability line of rotating equilibrium configurations (Takami et al. 2011; Weih et al. 2018) (red solid line in the inset) and is not straightforward to compute. However, it is well approximated by the turningpoint line (Friedman et al. 1988), which is far simpler to compute and has a maximum value, $M_{\max }$, representing the largest mass for configurations on the mass-shedding limit (dashed black line in the inset). More importantly, both the maximum mass and the critical masses are related to $M_{\text {Tov }}$ through universal relations, i.e., $M_{\text {crit }} \leq M_{\text {max }} \simeq 1.2 M_{\text {Tov }}$ (Breu \& Rezzolla 2016); $M_{\max }$ is marked with black a filled square in the inset. In essence, therefore, for the primary to be gravitationally stable, its position in the $(\tilde{\chi}, q)$ plane will have to be in the green-shaded region; outside this region, the primary is either unstable or differentially rotating.

As a result, for any value of the dimensionless spin $\tilde{\chi}$, the green-shaded area is bounded from below by a "critical mass ratio"

$q_{\mathrm{crit}}\left(\tilde{\chi}, M_{\mathrm{tot}}\right):=\frac{M_{\mathrm{tot}}}{M_{\mathrm{crit}}(\tilde{\chi})}-1$,

set only by the critical mass at that dimensionless spin an by the total mass of the binary. Note that after some (reasonable) assumption about the spin distribution in the binary, expression (2) can effectively be cast only in terms of the dimensionless spin of the primary $\chi_{1}:=S_{1} / m_{1}^{2}$. This follows directly from the definition of the dimensionless spin

$\tilde{\chi}:=\frac{m_{1} \chi_{1}+m_{2} \chi_{2}}{m_{2}+m_{1}}=\frac{\chi_{1}}{1+q}\left(1+q \frac{\chi_{2}}{\chi_{1}}\right)=\frac{\chi_{1}}{1+q}\left(1+\frac{1}{q} \frac{S_{2}}{S_{1}}\right)$,

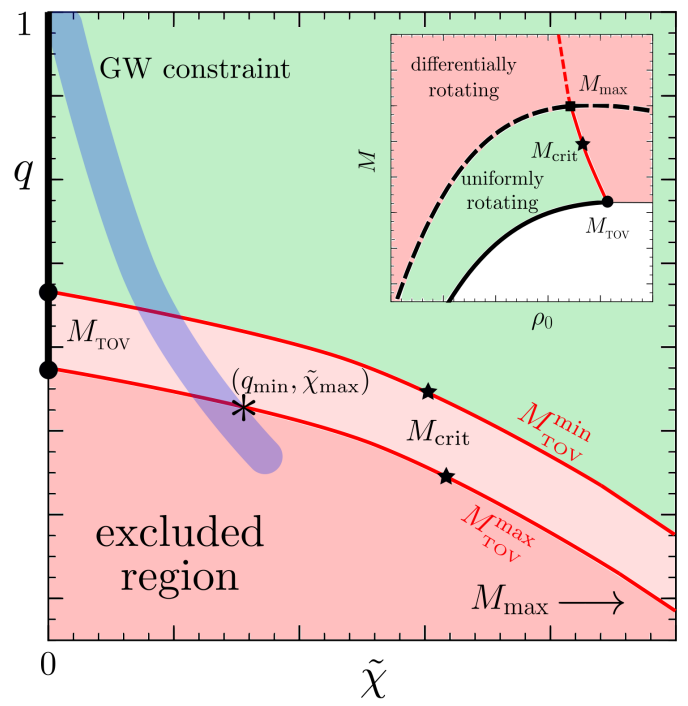

Figure 1. Broadbrush picture of how to constrain the mass ratio $q$ and effective dimensionless spin $\tilde{\chi}$ of a high-mass merger. The inset reports the same configurations in terms of the mass of the star $M$ and its central density $\rho_{0}$. A detection of a merger (blueshaded area) set constraints on models that are gravitationally stable (green-shaded area) and on models that are gravitationally unstable (red-shaded area; see Sec. 2 for details).

so that an estimate of the ratio of the two components' spin, $\chi_{1} / \chi_{2}\left(S_{2}\right.$ and $\chi_{2}$ are the spin and dimensionless spin of the secondary, respectively) allows one to express $q_{\text {crit }}=q_{\text {crit }}\left(\chi_{1}, M_{\text {tot }}\right)$.

Due to the uncertainty in the EOS of nuclear matter, this exclusion boundary is not known exactly and is therefore represented by the light-red shaded area in Fig. 1. However, by computing this boundary for a large dataset of $O\left(10^{6}\right)$ EOSs, we can find a lower limit below which an excluded region can be defined safely. This lower limit is given by the EOSs with the highest $M_{\text {TOV }}$, i.e., $M_{\text {TOV }}^{\max }$ for a given dimensionless spin, thus defining the minimum critical mass ratio $q_{\mathrm{min}}:=q_{\mathrm{crit}}\left(\tilde{\chi}, M_{\mathrm{tot}}, M_{\mathrm{TOV}}^{\mathrm{max}}\right)$. In turn, $q_{\mathrm{min}}$ provides an upper limit on $\tilde{\chi}$, i.e., $\tilde{\chi}_{\max }$ (asterisk in Fig. 1).

In what follows, we apply the general considerations made so far to the specific case of GW190425 and discuss how we can set new limits on $M_{\text {TOV }}$ with future detections of high-mass binaries.

\section{UNIVERSAL RELATIONS AND FITS}

While the properties of neutron stars depend on their composition and hence on the still unknown EOS, some of their bulk properties, such as the moment of inertia or the quadrupole moment, have been shown to obey quasiuniversal relations (see Yagi \& Yunes 2017 for a review, but also Haskell et al. 2014 and Doneva et al. 2014 for examples of when these relations break). The appeal of quasiuniversality is that they allow to make predictions and place constraints independent of the uncertainty in the EOS. For this reason, we will make use of them in order to compute a solid estimate of the exclusion line (2). 
According to Breu \& Rezzolla (2016), the critical mass $M_{\text {crit }}$ (i.e., the mass along the red solid line in the inset of Fig. 1) fulfils a quasi-universal relation

$M_{\text {crit }}\left(\chi_{\text {crit }}, \chi_{\mathrm{kep}}, M_{\mathrm{TOV}}\right):=M_{\mathrm{TOV}}\left(1+a_{1}\left(\frac{\chi_{\text {crit }}}{\chi_{\text {Kep }}}\right)^{2}+a_{2}\left(\frac{\chi_{\text {crit }}}{\chi_{\mathrm{Kep}}}\right)^{4}\right)$,

where $a_{1}=0.132, a_{2}=0.071$ and $\chi_{\text {Kep }}$ is the largest possible dimensionless spin for the primary star at the massshedding limit. At this limit, $M_{\text {crit }}\left(\chi_{\text {crit }}=\chi_{\text {Kep }}\right)=: M_{\max }=$ $(1.203 \pm 0.022) M_{\text {TOV }}$ and $\chi_{\text {Kep }}$ is related to the compactness $C_{\mathrm{TOV}}:=M_{\mathrm{TOV}} / R_{\mathrm{TOV}}$, where $R_{\mathrm{TOV}}$ is the radius of the nonrotating neutron star configuration with the maximum mass $M_{\text {Tov }}$. In particular, starting from the analysis of Breu \& Rezzolla (2016) and Koliogiannis \& Moustakidis (2020), Shao et al. (2020) found that for large compactnesses the dimensionless Keplerian angular momentum shows a weak but linear dependence on $\sqrt{C}$. We therefore model this behaviour as

$\chi_{\mathrm{Kep}} \simeq \frac{\alpha_{1}}{\sqrt{C_{\mathrm{TOV}}}}+\alpha_{2} \sqrt{C_{\mathrm{Tov}}}$,

where the first term was initially suggested by Breu \& Rezzolla (2016) and the second one by Shao et al. (2020). A direct fit to the data of Breu \& Rezzolla (2016) and Rezzolla et al. (2018) yields $\alpha_{1}=0.045 \pm 0.021$ and $\alpha_{2}=1.112 \pm 0.072$. We should note that since $\alpha_{1} \ll \alpha_{2}$, Shao et al. 2020 have taken $\alpha_{1}=0$ as a first approximation, although $\alpha_{1} \neq 0$ is important to recover the Newtonian limit. Furthermore, because the dependence is weak and the scatter in the data large, setting $\chi_{\text {Kep }} \simeq$ const. $=0.682$ provides a remarkably good approximation. In addition, it hints the intriguing suggestion that - in analogy to black holes - a quasi-universal upper limit exists for the dimensionless spin of uniformly rotating compact stars.

By inverting Eq. (4), we can write the critical dimensionless spin as

$\chi_{\text {crit }}=\chi_{\text {kep }}\left(C_{\mathrm{TOV}}\right) \sqrt{-\frac{a_{1}}{2 a_{2}}+\sqrt{\frac{a_{1}^{2}}{4 a_{2}^{2}}+\frac{1}{a_{2}}\left(\frac{M_{\mathrm{crit}}}{M_{\mathrm{TOV}}}-1\right)} .}$

Stated differently, Eq. (6) provides an analytic expression for the dimensionless spin of stellar models along the stability line of uniformly rotating configurations (red solid line in Fig. 1). This quantity is only a function of $M_{\text {Tov }}$ and $R_{\text {TOV }}$, which are, however, unknown since a precise EOS of neutron stars is still undetermined. Furthermore, since $\chi_{\text {crit }} \propto C_{\text {TOV }}^{1 / 2}$, to derive a lower limit for the dimensionless spin $\chi_{\text {crit }}$ needed to support a given critical mass $M_{\text {crit }}$, we obviously need to provide a lower bound for $C_{\mathrm{TOV}}$, namely, $C_{\mathrm{TOV}}^{\mathrm{min}}$.

Luckily, the multimessenger detection of GW170817 has provided several constraints on the EOS and the corresponding radii (e.g., Annala et al. 2018; Most et al. 2018; Abbott et al. 2018; Weih et al. 2019b) and maximum mass (Margalit \& Metzger 2017; Rezzolla et al. 2018; Ruiz et al. 2018; Shibata et al. 2019) of neutron stars. In particular, exploiting this detection, Most et al. (2018) and Weih et al. (2019b) have built a large dataset of $O\left(10^{6}\right)$ EOSs satisfying recent constraints from chiral effective-field theory (Drischler et al. 2016), tidal deformabilities (Radice et al. 2018; Abbott et al. 2018; Kiuchi et al. 2019), i.e., $280<\Lambda_{1.4}<560$ for a $1.4 M_{\odot}$ star, and the maximum mass of nonrotating neutron stars (Margalit \& Metzger 2017; Rezzolla et al. 2018; Ruiz et al. 2018; Shibata et al. 2019), i.e., $1.97<M_{\text {Tov }} / M_{\odot} \lesssim 2.3$. We remark that these upper bounds on the maximum mass assume the formation of a black hole, which is plausible given the follow-up observations consistent with a structured jet (see, e.g., Hajela et al. 2019), or the constraints on the overproduction of blue ejected matter (see, e.g., Gill et al. 2019). However, if a black hole was not formed in GW179817, the maximum mass could be $M_{\text {Tov }} \gtrsim 2.4 M_{\odot}$ (Ai et al. 2019).

Using the before mentioned dataset, we can find (and fit) an lower bound for $C_{\mathrm{TOV}}^{\mathrm{min}}$ in terms of the maximum mass for nonrotating stars $M_{\mathrm{TO}}$ as the latter varies across the various EOSs. In this way, we derive

$\sqrt{C_{\mathrm{TOV}}^{\min }}=c_{1} M_{\mathrm{TOV}}+c_{2} M_{\mathrm{TOV}}^{2}+c_{3} M_{\mathrm{TOV}}^{3}$,

where $c_{1}=0.482, c_{2}=-0.174, c_{3}=0.027$. Note that $(7)$ can also be seen as a fit to the upper bound for $R_{\text {TOV }}$ and that, using as upper bound for the maximum mass the value $M_{\mathrm{TOV}}^{\max }=2.3 M_{\odot}$, we obtain $C_{\mathrm{Tov}}^{\min }\left(M_{\mathrm{TOV}}^{\max }\right)=0.265$, corresponding to a radius $R_{\mathrm{TOV}}^{\max }=12.81 \mathrm{~km}$.

At this point, using all the EOSs in our datasets, we can define the maximum mass that a rotating star with critical dimensionless spin can support when $\chi_{\text {kep }}=\chi_{\text {kep }}\left(C_{\mathrm{Tov}}^{\min }\right)=$ : $\chi_{\text {kep }}^{\min }$ and its mass is $M_{\text {crit }}^{\max }=M_{\text {crit }}\left(\chi_{\text {crit }}, \chi_{\text {kep }}^{\min }\left(M_{\text {TOV }}\right), M_{\text {TOV }}\right)$. To this scope, all we need to do is to insert Eq. (5) in Eq. (4), with $C_{\mathrm{TOV}}=C_{\mathrm{TOV}}^{\mathrm{min}}$ as given by Eq. $(7)^{1}$.

\section{APPLiCATION TO GW190425 AND FUTURE EVENTS}

We now apply the methodology illustrated so far to the specific case of GW190425 and in this way derive constraints on its mass ratio $q$ and dimensionless spin $\tilde{\chi}$. As outlined in Sec. 2, we need to compute the minimal mass ratio $q_{\text {crit }}$ using Eq. (2), together with the expression for the critical mass $M_{\text {crit }}$ given by Eq. (4). Additionally, we need to provide an ansatz for the relation between the two spins $S_{1}, S_{2}$, in order to express $\tilde{\chi}=\tilde{\chi}\left(\chi_{1}, q\right)$. We therefore consider four different plausible aligned $(\uparrow \uparrow)$ or antialigned $(\uparrow \downarrow)$ spin configurations, although it is straightforward to consider other cases as a function of $q$ :

$$
\begin{array}{llll}
\uparrow \uparrow a: & S_{2}=S_{1} & \longleftrightarrow & \tilde{\chi}=\chi_{1} / q \\
\uparrow \uparrow_{b}: & S_{2}=q S_{1} & \longleftrightarrow & \tilde{\chi}=2 \chi_{1} /(1+q) \\
\uparrow \downarrow_{a}: & S_{2}=-S_{1} & \longleftrightarrow & \tilde{\chi}=\chi_{1}(1-1 / q) /(1+q) \\
\uparrow \downarrow_{b}: & S_{2}=-q^{2} S_{1} & \longleftrightarrow & \tilde{\chi}=\chi_{1}(1-q) /(1+q)
\end{array}
$$

Note that $\tilde{\chi}=0$ for $S_{2}=-q S_{1}$ or, equivalently, $\chi_{2}=-\chi_{1} / q$, and that our choices of $\tilde{\chi}$ are deliberately kept simple to illustrate the power of the method. In particular, all of them are constructed such that $\tilde{\chi}=0$ when $\chi_{1}=0$; more elaborate choices can trivially be incorporated into our approach, but are beyond the scope of this Letter.

Using Eq. (4) for the definition of $M_{\text {crit }}^{\max }$, we can then

1 Note that we use $C_{\mathrm{TOV}}^{\min }$ and not $C_{\mathrm{TOV}}$ since we are searching for an upper limit and $M_{\text {crit }}\left(C_{\text {TOV }}^{\min }, M_{\text {Tov }}, \chi_{\text {crit }}\right) \geq M_{\text {crit }}\left(C_{\text {TOV }}, M_{\text {Tov }}, \chi_{\text {crit }}\right)$ in the dataset. 


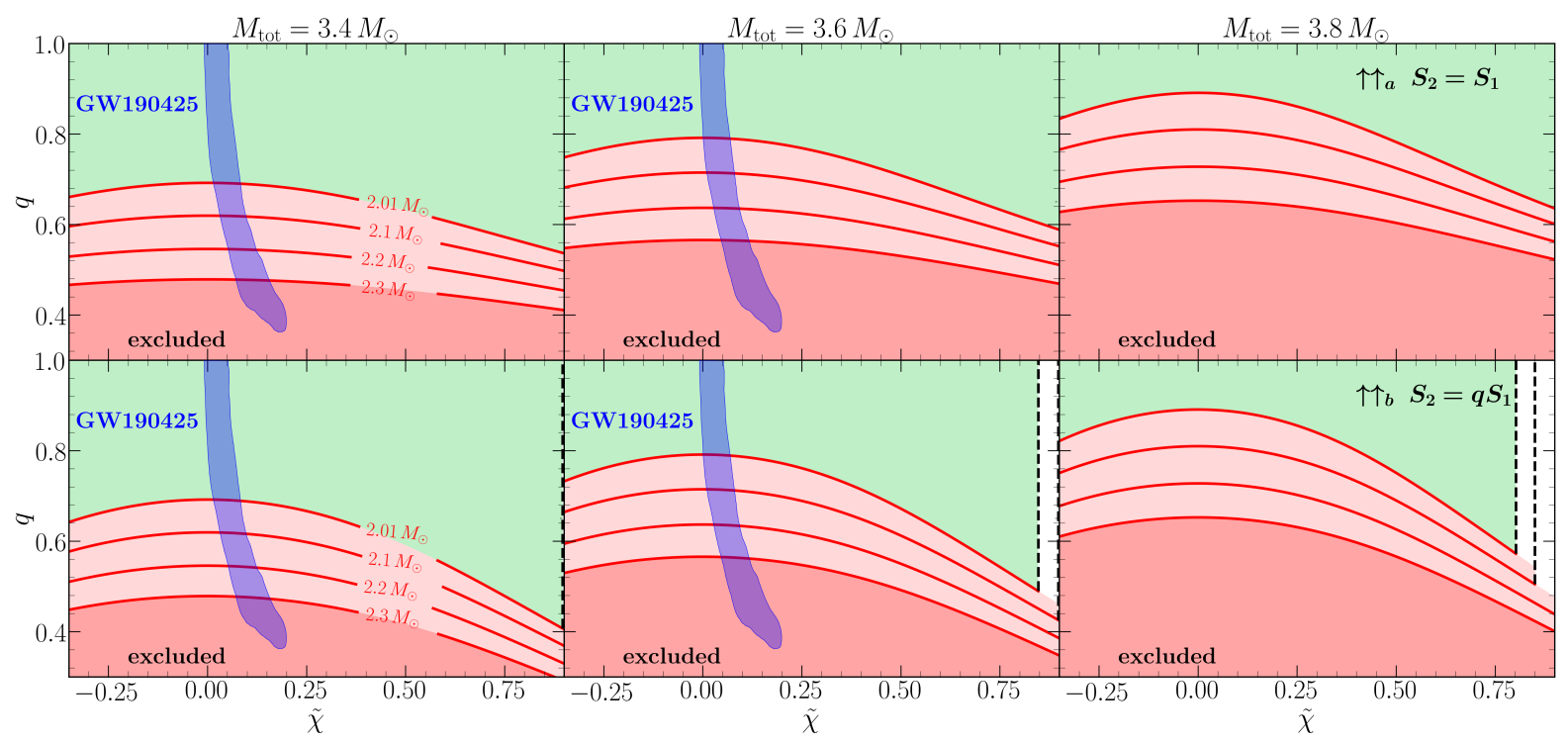

Figure 2. Exclusion lines on the binary mass ratio $q$ and mass weighted spin $\tilde{\chi}$ for high-mass binary neutron star mergers with total masses $\boldsymbol{M}_{\mathrm{tot}} / \boldsymbol{M}_{\odot}=(3.4,3.6,3.8)$ and aligned spins. Similar to Fig. 1, the green areas represent the allowed parameter space of stable models, while the dark-red (excluded) areas do not contain stable binary configurations. The exclusion line is determined by the maximum mass $M_{\mathrm{TOV}}$, whose uncertainty translates to the light-red shaded areas. The observationally inferred parameter range for GW190425 is indicated by the blue-shaded area.

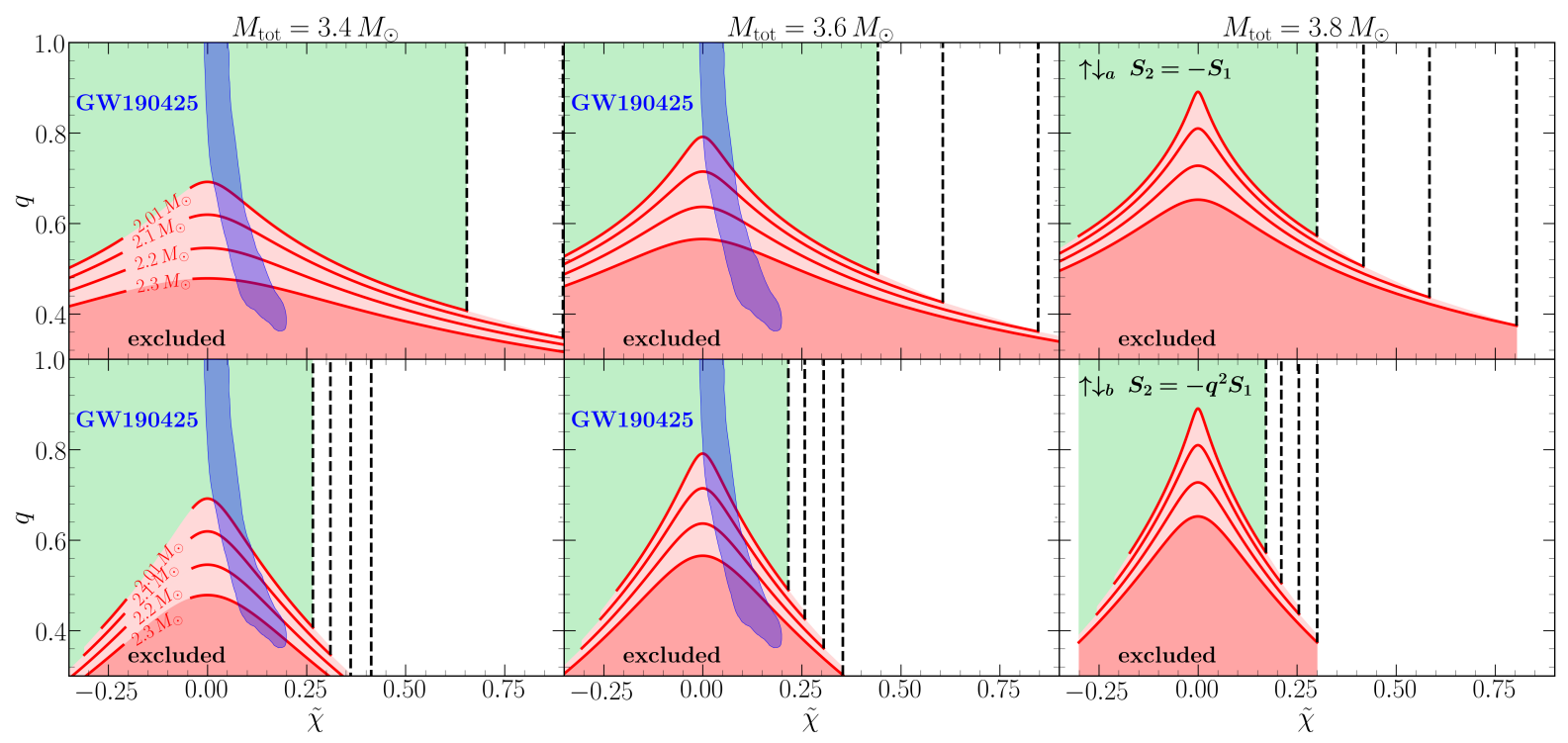

Figure 3. Same as Fig. 2 but for antialigned spin configurations. The vertical black dashed line refers to the models on the mass-shedding limit (cf., Fig. 1); the properties of the models beyond these lines depend on the EOS and could either be stable or unstable.

compute the allowed range in mass ratios $q>q_{\text {crit }}$ as given by Eq. (2). The resulting parameter space in the $(\tilde{\chi}, q)$ plane is shown in Figs. 2 and 3 using the same colourcoding and line conventions introduced in Fig. 1. Overall, we show results for three different values of the total mass, i.e., $M_{\text {tot }}=3.4,3.6$ and $3.8 M_{\odot}$ (left, middle and right panels respectively), where $3.4 M_{\odot}$ applies most closely to GW190425. Clearly, the red solid lines represent the contours separating the allowed/excluded regions for different choices of $M_{\mathrm{TOV}} \in[2.01 ; 2.3] M_{\odot}$.

Concentrating first on the case of GW190425 with highspin prior $\tilde{\chi}<0.95$ (Abbott et al. 2020) (blue-shaded region, left panels in Figs. 2 and 3), we can appreciate that the constraints from GW190425 do fall in excluded regions. Stated differently, below a certain mass ratio, no binary configurations are possible in which the stars can be supported through rotation and are compatible with the observations. This threshold does depend on the choice of spin configuration, although in the specific case of GW190425 only weakly, since the inferred dimensionless effective spins are small, i.e., $\tilde{\chi}<0.2$. Furthermore, when considering different values of the maximum mass $M_{\text {TOV }}$, this threshold is present even when taking an upper value of $M_{\text {TоV }} \simeq 2.3 M_{\odot}$, as suggested by Rezzolla et al. (2018) and Shibata et al. (2019). 
Overall, in the case of GW190425 we can conclude that mass ratios $q \lesssim 0.5$ are robustly excluded across the different choices of spin configurations. This exclusion is almost independent of the value of $\tilde{\chi}<0.2$, since the mass weighted spin is too low for rotation to significantly increase the supported maximum mass and thus $M_{\text {crit }} \simeq M_{\text {Tov }}$. To be more precise, we find that $q_{\min } \gtrsim 0.46(0.38)$ for aligned (antialigned) configurations. Similarly, we can set upper limits on the dimensionless spin, with $\tilde{\chi}_{\max } \lesssim 0.16(0.20)$ for aligned (antialigned) configurations; the corresponding constraints when considering a mass of $3.6 M_{\odot}$ for GW190425 are: $q_{\min } \gtrsim 0.56(0.46)$ and $\tilde{\chi}_{\max } \lesssim 0.12(0.17)$. We note that Foley et al. (2020) have recently presented similar constraints for GW190425 applying a different line of arguments. In particular, assuming a Gaussian distribution of $M_{\mathrm{TOV}}=2.1 \pm 0.12 M_{\odot}$ at $2 \sigma$ and prior of $\chi_{1}<0.4$ chosen to include stellar models computed with a selection of EOSs, they provide a lower limit $q>0.53$ at $3 \sigma$ for GW190425, which is in good agreement with our findings.

We next discuss how these results change when considering hypothetical higher-mass binaries (i.e., $M_{\text {tot }}=$ 3.6,3.8 $M_{\odot}$ in the mid and right panels in Figs. 2 and 3), noting that quite generically the red-shaded bands of rotationally unstable models, and thus the exclusion regions, shift upwards [cf., Eq. (2)]. For aligned configurations, the rotational corrections are very small (the red solid lines are almost horizontal in the middle and right panels of Fig. 3), so that for $M_{\mathrm{TOV}}=2.3 M_{\odot}$ we can set nonrotating (i.e., $\tilde{\chi}=0$ ) cutoffs of $q \gtrsim 0.4$ and $q \gtrsim 0.5$ for the aligned cases $\uparrow \uparrow a$ and $\uparrow_{b}$, respectively. In the case of misaligned spins, on the other hand, the spin effects are much more pronounced and the exclusion regions move up significantly. As a result, we can set nonrotating cutoffs of $q \gtrsim 0.56$ and $q \gtrsim 0.65$ for the antialigned cases $\uparrow \downarrow_{a}$ and $\uparrow \downarrow_{b}$, respectively. Interestingly, even for small dimensionless spins, the degeneracy in $M_{\text {Tov }}$ is removed with increasing spin and slightly more asymmetric binaries with $q<0.4$ begin to be allowed, albeit at very high individual spins.

Most prominently, shown as white-shaded ares in Fig. 3 are the early terminations of the regions of rotationally supported neutron stars. This follows directly from the upper limit of $\chi_{\mathrm{Kep}} \geq \chi_{\text {crit }}$ on the dimensionless spin of rotating stars with maximum masses $M_{\text {crit }}$. Since the antialigned cases have a partial cancellation of the individual component spins, $\chi_{1}$ will reach $\chi_{\text {Kep }}$ already for $\tilde{\chi}<0.6$. As a trivial consequence this also implies that there is an exclusion region for $\chi_{1}>\chi_{\text {Kep }}$ and hence also an upper limit on $\tilde{\chi}<\tilde{\chi}_{\text {kep }}$, as marked in Fig. 3 with vertical black dashed lines. Note that the stability properties of the models beyond these lines depend on the EOS and could lead to either green- or redshaded regions and to complex boundaries. For simplicity, and because it is irrelevant for our discussion here, we simply use a white shading.

\section{CONCLUSIONS AND OUTLOOK}

We have investigated how the properties of high-mass neutron-star mergers, such as the mass ratio $q$ and the dimensionless spin $\tilde{\chi}$, can be constrained by combining our current knowledge of the EOS of nuclear matter and universal relations. In particular, combining universal relations

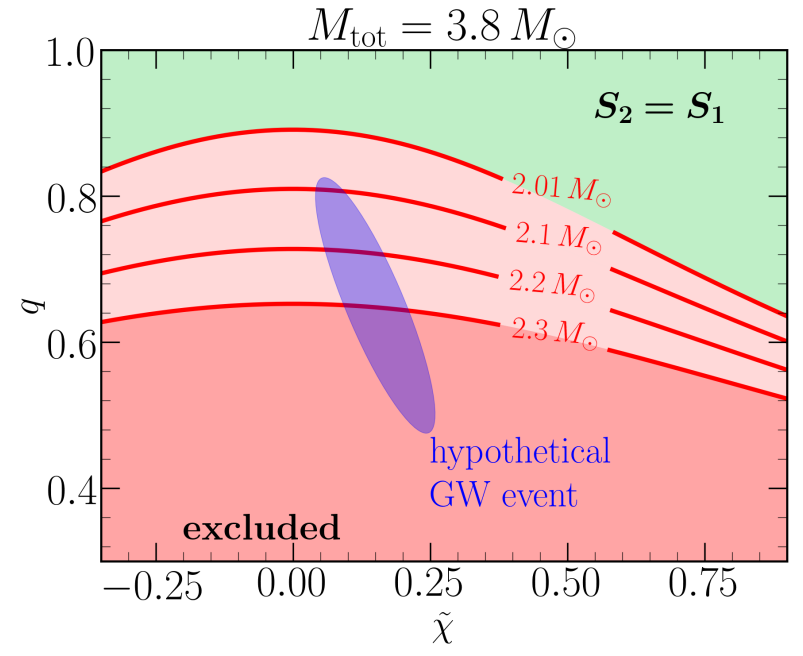

Figure 4. Same as Fig. 2 but for a hypothetical future gravitational event. A large total mass and a large asymmetry in the mass ratio would a provide stringent lower bound on $M_{\mathrm{TOV}}$.

for the critical mass of uniformly rotating neutron stars $M_{\text {crit }}$ (Breu \& Rezzolla 2016) with a large dataset of physically plausible EOSs constrained by the GW170817 event (Most et al. 2018; Weih et al. 2019b), we have been able to derive a lower limit for the mass ratio $q_{\min }$ and an upper limit on the dimensionless spin $\tilde{\chi}_{\text {max }}$. While these limits depend on the spin configuration of the binary and on the assumed maximum mass for nonrotating configurations $M_{\text {Tov }}$, we could set constraints on the properties of the gravitational-wave event GW190525 after considering four representative cases of aligned and antialigned binaries and an upper limit of $M_{\text {TоV }}=2.3 M_{\odot}$. In this way, we have concluded that $q_{\min } \geq 0.48$ and $\tilde{\chi}_{\max } \leq 0.16$ for GW190425, thus ruling out some of the most extreme binary configurations. Future refinements on the value of $M_{\mathrm{TOV}}$ will further constrain these limits.

As an interesting outlook, we finally illustrate the hypothetical scenario of a neutron-star merger in which the observations - either from the gravitational-wave detection or from the electromagnetic counterpart (i.e., amount of mass ejection, estimated life time, etc.) - set an upper limit on the mass ratio, $q_{\max }$. This is illustrated in Fig. 4 , which is the same as the top right panel of Fig. 2, and where we have considered a hypothetical high-mass merger with total mass $M_{\mathrm{tot}}=3.8 M_{\odot}$, which has set a constraint in the $(\tilde{\chi}, q)$ plane (blue-shaded area). Should this be the case, it would then be trivial to place a lower bound on $M_{\text {Tov }}$ from the intersection of the detection band with the contour lines shown in Fig. 4. More formally, given an upper value for the mass ratio $q_{\max }$ - and almost independently of the estimate for $\tilde{\chi}$ - it would be possible to invert numerically Eq. (4) and find therefore a strict lower limit for $M_{\mathrm{TOV}}$. In the limit of $\tilde{\chi} \rightarrow 0$, this will have the trivial solution $M_{\mathrm{Tov}}>M_{\mathrm{tot}} /(1+q)$.

\section{ACKNOWLEDGEMENTS}

It is a pleasure to thank Yizhong Fan and L. Jens Papenfort for useful discussions and comments. ERM and LRW acknowledge support through HGS-HIRe. Support comes in 
part from HGS-HIRe for FAIR; the LOEWE-Program in HIC for FAIR; "PHAROS", COST Action CA16214 European Union's Horizon 2020 Research and Innovation Programme (Grant 671698) (call FETHPC-1-2014, project ExaHyPE); the ERC Synergy Grant "BlackHoleCam: Imaging the Event Horizon of Black Holes" (Grant No. 610058);

\section{REFERENCES}

Abbott B. P., et al., 2018, Physical Review Letters, 121, 161101 Abbott B. P., et al., 2020, Astrophys. J. Lett., 892, L3

Ai S., Gao H., Zhang B., 2019, arXiv e-prints, p. arXiv:1912.06369 Annala E., Gorda T., Kurkela A., Vuorinen A., 2018, Phys. Rev. Lett., 120, 172703

Breu C., Rezzolla L., 2016, Mon. Not. R. Astron. Soc., 459, 646

Coughlin M. W., et al., 2020, Mon. Not. R. Astron. Soc., 492, 863

De S., Finstad D., Lattimer J. M., Brown D. A., Berger E., Biwer C. M., 2018, Physical Review Letters, 121, 091102

Doneva D. D., Yazadjiev S. S., Stergioulas N., Kokkotas K. D., 2014, Astrophys. J. Letters, 781, L6

Drischler C., Carbone A., Hebeler K., Schwenk A., 2016, Phys. Rev. C, 94, 054307

Farrow N., Zhu X.-J., Thrane E., 2019, Astrophys. J., 876, 18

Foley R. J., Coulter D. A., Kilpatrick C. D., Piro A. L., RamirezRuiz E., Schwab J., 2020, arXiv e-prints, p. arXiv:2002.00956

Friedman J. L., Ipser J. R., Sorkin R. D., 1988, Astrophys. J., 325,722

Gill R., Nathanail A., Rezzolla L., 2019, Astrophys. J., 876, 139

Hajela A., et al., 2019, Astrophys. J. Lett., 886, L17

Han M.-Z., Tang S.-P., Hu Y.-M., Li Y.-J., Jiang J.-L., Jin Z.-P., Fan Y.-Z., Wei D.-M., 2020, arXiv e-prints, p. arXiv:2001.07882

Haskell B., Ciolfi R., Pannarale F., Rezzolla L., 2014, Mon. Not. R. Astron. Soc., 438, L71

Kiuchi K., Kyutoku K., Shibata M., Taniguchi K., 2019, Astrophys. J., 876, L31

Koeppel S., Bovard L., Rezzolla L., 2019, Astrophys. J. Lett., 872, L16

Koliogiannis P. S., Moustakidis C. C., 2020, Phys. Rev. C, 101, 015805

Kyutoku K., Fujibayashi S., Hayashi K., Kawaguchi K., Kiuchi K., Shibata M., Tanaka M., 2020, arXiv e-prints, p. arXiv:2001.04474

Margalit B., Metzger B. D., 2017, Astrophys. J. Lett., 850, L19

Montaña G., Tolós L., Hanauske M., Rezzolla L., 2019, Phys. Rev. D, 99, 103009

Most E. R., Weih L. R., Rezzolla L., Schaffner-Bielich J., 2018, Phys. Rev. Lett., 120, 261103

Radice D., Perego A., Zappa F., Bernuzzi S., 2018, Astrophys. J. Lett., 852, L29

Rezzolla L., Most E. R., Weih L. R., 2018, Astrophys. J. Lett., 852, L25

Romero-Shaw I. M., Farrow N., Stevenson S., Thrane E., Zhu X.-J., 2020, arXiv e-prints, p. arXiv:2001.06492

Ruiz M., Shapiro S. L., Tsokaros A., 2018, Phys. Rev. D, 97, 021501

Safarzadeh M., Ramirez-Ruiz E., Berger E., 2020, arXiv e-prints, p. arXiv:2001.04502

Shao D.-S., Tang S.-P., Sheng X., Jiang J.-L., Wang Y.-Z., Jin Z.-P., Fan Y.-Z., Wei D.-M., 2020, Phys. Rev. D, 101, 063029

Shibata M., Zhou E., Kiuchi K., Fujibayashi S., 2019, Phys. Rev. D, 100, 023015

Takami K., Rezzolla L., Yoshida S., 2011, Mon. Not. R. Astron. Soc., 416, L1

Tews I., Carlson J., Gandolfi S., Reddy S., 2018, Astrophys. J., 860,149
The LIGO Scientific Collaboration The Virgo Collaboration 2017, Phys. Rev. Lett., 119, 161101

Weih L. R., Most E. R., Rezzolla L., 2018, Mon. Not. R. Astron. Soc., 473, L126

Weih L. R., Most E. R., Rezzolla L., 2019a, EOS library, doi:10.5281/zenodo.3260991, https://doi.org/10. 5281/zenodo. 3260991

Weih L. R., Most E. R., Rezzolla L., 2019b, Astrophys. J., 881, 73

Yagi K., Yunes N., 2017, Phys. Rep., 681, 1 